\title{
MPCSToken: Smart Contract Enabled Fault-Tolerant Incentivisation for Mobile P2P Crowd Services
}

\author{
Fengrui Shi*, Zhijin Qin ${ }^{\dagger}$, Di Wu ${ }^{\ddagger}$, and Julie A. McCann* \\ * \{fengrui.shi14, j.mccann\}@imperial.ac.uk, Department of Computing, Imperial College London, UK \\ ${ }^{\dagger}$ zhijin.qin@lancaster.ac.uk, School of Computing and Communications, Lancaster University, UK

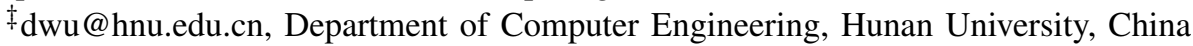

\begin{abstract}
Mobile peer to peer (P2P) networks offer a huge potential for distributed mobile P2P crowd services (MPCS), which enable data and computational tasks to be offloaded and executed directly between mobile devices. Similar to centralised mobile crowd services, such as mobile crowdsensing, incentivisation mechanisms are core to encouraging mobile users to participate in MPCS systems. However, due to the impact of task execution failures and unreliable behaviours of mobile users (particularly task requesters), it is a daunting task to design and implement an incentivisation mechanism to cater for the needs of MPCS systems. In this paper, we propose a fault-tolerant incentivisation mechanism (FTIM) for MPCS systems. With conditional payment strategies, FTIM is proven to accommodate the requirements of two important application scenarios by achieving mechanism properties such as incentive compatibility, economic efficiency, individual rationality, and weak budget balance. Moreover, to tackle the practical challenges in implementing FTIM in the real world, we design a MPCSToken smart contract to facilitate its service auction, task execution and payment settlement process. We implement the MPCSToken contract on Ethereum blockchain. Both real-world experiment and simulation results show that the system is cost effective for deployments and improves the overall mobile users' utility by exploring the opportunities offered by MPCS.
\end{abstract}

\section{INTRODUCTION}

With the proliferation of mobile devices, mobile crowd participants have become one of the important providers for data and services. For example, mobile crowdsensing [1], [2] is a paradigm that relies on the data collection (sensing) capability of mobile devices to assist various tasks such as floorplan construction [3], [4], traffic management [5], and environmental monitoring [6]. The crowdsensing based approach usually relies on a centralised cloud server to allocate tasks and provide incentives to the participating mobile users. The recent development of device to device (D2D) communications enables mobile peer to peer (P2P) crowd service (MPCS) systems, in which data offloading and computation tasks can be provided in a fully distributed fashion between several mobile devices in the local network formed by Bluetooth or WiFi Direct [7]-[11]. Compared to centralised mobile crowd service systems, in which the cloud server is the service requester and the mobile users are service providers (usually called crowd workers or workers), both the roles of service providers (workers) and requesters are undertaken by mobile users in MPCS systems. Fig. 11 illustrates several example services that can be provided in MPCS systems. These examples

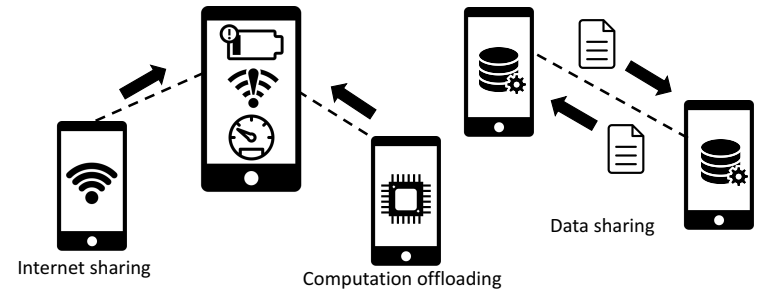

Fig. 1. Overview of mobile P2P crowd services.

include not only P2P data sharing and offloading [7], [8], but also various application specific computation tasks [9], [10].

Mobile users may be selfish and not willing to participate in MPCS systems without benefits. Therefore, an incentivisation mechanism is the key to the success of MPCS systems. Centralised mobile crowd service systems typically employ mechanism design theory to incentivise mobile crowd workers, in which a payment strategy is adopted by the cloud server to offer crowd workers monetary rewards in return for their data and services [11]-[15]. To ensure that the payment strategy is executed faithfully, it is essential that the cloud server is trustworthy. However, in MPCS systems, the execution uncertainty of tasks in mobile environments and the fact that service requesters, being mobile users, may also be selfish and untrustworthy, bring many complexities and challenges in designing and implementing incentivisation mechanisms:

a) Uncertainty of service delivery/task execution: Due to unstable network conditions and mobile device capacities, requested services and tasks may be subject to execution failures. For example, Internet sharing services and computation tasks are affected by the network connectivity and the CPU capacity of mobile devices respectively. Therefore, it is essential to take service delivery and task execution uncertainty into consideration when designing incentivisation mechanisms for MPCS systems.

b) Untruthful service requesters: Mobile users may be selfish and strategic, which makes their behaviours essentially untruthful. Although mechanisms have been proposed to solve such issues [13], [15], [16], they are not suitable for MPCS systems due to the fact that both service providers and mobile workers in MPCS systems can be untruthful. As a result, a payment strategy ensuring truthful reporting of private information by both service requesters and mobile crowd workers is required by the incentivisation mechanism. 
c) Difficulty of enforcing payment: Services in MPCS systems are provided in an off-line D2D fashion and their execution and delivery results are difficult to be verified. Moreover, the task execution and payment processes are carried out in an opportunistic and distributed fashion. Both factors make the payment difficult to be enforced since any counterparty can vanish without paying for the services or refuse to offer services that have already been paid for. To ensure the practicality for an incentivisation mechanism in real-world MCPS systems, a payment settlement mechanism must be built into the system to mitigate this risk.

To tackle these challenges, this paper designs a fault-tolerant incentivisation mechanism (FTIM) with micropayment based settlements. Moreover, the proposed mechanisms are implemented as a blockchain smart contract. To the best of our knowledge, this is the first work that designs and implements an incentivisation mechanism for MPCS systems, particularly using blockchain and smart contract technologies. The detailed contributions of this paper include:

- FTIM, a fault-tolerant incentivisation mechanism based on payment strategies conditional on task execution results, is proposed. We highlight two application scenarios for MPCS and prove that FTIM provides the scenarios with essential properties including incentive compatibility (IC), individual rationality (IR) and economic efficiency (EE), and weak budget balance (weak BB).

- FTIM is deployed as a smart contract named MPCSToken contract to show the practicality of the proposed mechanisms. Moreover, we build a micropayment mechanism based on blockchain payment channels into the MPCSToken contract to avoid bulky payments and mitigate payment settlement risks.

- We implement the MPCSToken contract on Ethereum blockchain and evaluate its performance in terms of users' utility and blockchain cost with both real-world experiments and simulations. Evaluation results indicate that FTIM greatly improves mobile users' utility in facilitating MPCS, and the MPCSToken contract incurs low costs for deployment and execution on the blockchain.

The rest of the paper is organised as follows: Section II introduces the system model and formulates the incentivisation problem in MPCS systems. The proposed fault-tolerant incentivisation mechanism (FTIM) is presented in Section III Its payment strategies and mechanism properties are presented and proved, respectively. Section IV shows the structure of MPCSToken contract which implements FTIM. The implemented MPCSToken contract prototype, an experimental mobile offloading application and relevant evaluation results are shown and analysed in Section $\mathrm{V}$. Section $\mathrm{VI}$ and $\mathrm{VII}$ present related work and concludes this paper, respectively.

\section{Preliminaries}

\section{A. System Model}

We consider mobile users in an MPCS system with two types of roles - mobile service requesters and mobile crowd workers. A requester $\mathcal{R}$ is a mobile user who has a set of pending tasks $\mathcal{T}=\left\{\mathcal{T}_{j}\right\}, j \in\{1,2, \ldots, M\}$ and wishes to explore the possibility to offload these tasks to nearby mobile devices for execution services in order to reduce costs or save its own resources. In its proximity, $N$ workers $\mathcal{W}=\left\{\mathcal{W}_{i}\right\}$, $i \in\{1,2, \ldots, N\}$ are willing to execute the tasks on behalf of $\mathcal{R}$. In this paper, we assume that only one worker is executing task $\mathcal{T}_{j}$ for $\mathcal{R}$ simultaneously, since $\mathcal{R}$ is able to choose another worker if the task fails. In addition, a number of execution quality requirements $\mathcal{Q}_{j}$ are also set for $\mathcal{T}_{j}$.

Based on the quality requirements $\mathcal{Q}_{j}$, worker $\mathcal{W}_{i}$ is able to compute the probability $p_{i}^{j}$ of successfully fulfilling $\mathcal{Q}_{j}$ for $\mathcal{T}_{j}$, given their devices' real-time and projected resource capacity. In addition to $p_{i}^{j}, \mathcal{W}_{i}$ is also aware of its $\operatorname{cost} c_{i}^{j}$ for performing $\mathcal{T}_{j}$. Note that the $\operatorname{cost} c_{i}^{j}$ associated with executing $\mathcal{T}_{j}$ is always incurred even if $\mathcal{Q}_{j}$ is not satisfied because $\mathcal{W}_{i}$ and $\mathcal{R}$ can only obtain the quality results after executing $\mathcal{T}_{j}$. As for $\mathcal{R}$, if $\mathcal{T}_{j}$ is successfully executed by satisfying $Q_{j}$, it receives a positive value $V_{j}$, otherwise, no value is received by $\mathcal{R}$. Therefore, by allocating a task $\mathcal{T}_{j}$ to $\mathcal{W}_{i}$, the expected value that $\mathcal{R}$ receives is $v_{\mathcal{R}}=p_{i}^{j} \cdot V_{j}$ while the value received by worker $\mathcal{W}_{i}$ is $v_{i}^{j}=-c_{i}^{j}$. The overall expected utility (social welfare) for this transaction is thus $p_{i}^{j} \cdot V_{j}-c_{i}^{j}$.

In general, $p_{i}^{j}, c_{i}^{j}$ and $V_{j}$ are the private values of requester $\mathcal{R}$ and workers $\mathcal{W}$ and are called type vectors, denoted as $\boldsymbol{\theta}=\left(\theta^{\mathcal{R}}, \boldsymbol{\theta}^{\mathcal{W}}\right)$, where $\theta^{\mathcal{R}}$ is the type of $\mathcal{R}$ containing the value $V_{j}$ for tasks $\mathcal{T}_{j}$, and $\boldsymbol{\theta}^{\mathcal{W}}=\left\{\theta_{i}\right\}$ is the type vector of worker $\mathcal{W}_{i}$ with $p_{i}^{j}$ and $c_{i}^{j}$ for each task $\mathcal{T}_{j}$. As a convention, $-i$ is used to denote all workers except $\mathcal{W}_{i}$, and $\boldsymbol{\theta}^{\mathcal{W}}=\left(\theta_{i}, \boldsymbol{\theta}_{-i}\right)$ denotes the types of all workers. These private values may be different from the values declared by workers and requesters, which are represented by $\tilde{\theta}_{i}^{\mathcal{W}}\left(\tilde{p}_{i}^{j}, \tilde{c}_{i}^{j}\right)$ and $\tilde{\theta}^{\mathcal{R}}\left(\tilde{V}_{j}\right)$. The expected utility for worker $\mathcal{W}_{i}$ is defined as $u_{i}\left(\theta_{i}, \boldsymbol{\theta}_{-i}, \theta^{\mathcal{R}}\right)$ and $u_{\mathcal{R}}\left(\theta^{\mathcal{R}}, \boldsymbol{\theta}^{\mathcal{W}}\right)$ for the requester. In this paper, we consider a quasi-linear utility function $u\left(\theta_{i}, \boldsymbol{\theta}_{-i}, \theta^{\mathcal{R}}\right)=v\left(\theta_{i}, \boldsymbol{\theta}_{-i}, \theta^{\mathcal{R}}\right)+r$, where $r$ is the monetary transfer (payment) made by either $\mathcal{W}_{i}$ (denoted as $r_{i}$ ) or $\mathcal{R}$ (denoted as $r_{\mathcal{R}}$ ) to redistribute the overall utility $p_{i}^{j} \cdot V_{j}-c_{i}^{j}$. Thus, $u_{i}\left(\theta_{i}, \boldsymbol{\theta}_{-i}, \theta^{\mathcal{R}}\right)=-c_{i}^{j}-r_{i}$ and $u_{\mathcal{R}}\left(\theta^{\mathcal{R}}, \boldsymbol{\theta}^{\mathcal{W}}\right)=p_{i}^{j} \cdot V_{j}+r_{\mathcal{R}}$. A positive $r$ indicates receiving payment while a negative $r$ means making payment. Similar to $v\left(\theta_{i}, \boldsymbol{\theta}_{-i}, \theta^{\mathcal{R}}\right), r$ is also conditional on the task execution results. We denote $r^{+}$as the payment for a completed task and $r^{-}$for a failed task. An incentivisation mechanism $\mathcal{M}$ is designed by a third party entity, such as a system administrator or an application developer, to define the amount of $\left(r_{\mathcal{R}}^{+}, r_{\mathcal{R}}^{-}\right)$ and $\left(r_{i}^{+}, r_{i}^{-}\right)$conditional on the task execution results.

\section{B. Problem Formulation}

The objective of $\mathcal{M}$ is to maximise the expected utility by allocating task $\mathcal{T}_{j}$ to worker $\mathcal{W}_{i^{*}}$ according to:

$$
i^{*}=\underset{i \in \mathcal{W}}{\arg \max }\left\{\tilde{p}_{i}^{j} \cdot \tilde{V}_{j}-\tilde{c}_{i}^{j}\right\},
$$

This allocation strategy depends on the declared types of $\tilde{p}_{i}^{j}, \tilde{c}_{i}^{j}$ and $\tilde{V}_{j}$. Since both workers and requesters are acting strategically and trying to maximise their own benefits, the 
objective of $\mathcal{M}$ is to elicit truthful types from workers and requesters in order to achieve the maximal expected utility. A typical technique for achieving this is mechanism design [17], [18]. More specifically, if the value function $v\left(\theta_{i}, \boldsymbol{\theta}_{-i}, \theta^{\mathcal{R}}\right)$ is well defined (in this case, the value function is $p_{i}^{j} \cdot V_{j}$ for $\mathcal{R}$ and $-c_{i}^{j}$ for $\mathcal{W}_{i}$ ), designing an incentivisation mechanism $\mathcal{M}$ is in fact to design a payment strategy of $r_{\mathcal{R}}=\left(r_{\mathcal{R}}^{+}, r_{\mathcal{R}}^{-}\right)$and $r_{i}=\left(r_{i}^{+}, r_{i}^{-}\right)$.

A good incentivisation mechanism needs to offer certain properties. As for MPCS systems, the properties that mechanism $\mathcal{M}$ aims to achieve are listed as follows:

- Incentive Compatibility (IC): A mechanism is IC when mobile users (both requester and workers) maximise their utility by revealing their true types in equilibrium. Formally, it can be defined as $u_{i}\left(\theta_{i}, \boldsymbol{\theta}_{-i}, \theta^{\mathcal{R}}\right) \geq$ $u_{i}\left(\tilde{\theta}_{i}, \boldsymbol{\theta}_{-i}, \theta^{\mathcal{R}}\right)$ and $u_{\mathcal{R}}\left(\theta^{\mathcal{R}}, \boldsymbol{\theta}^{\mathcal{W}}\right) \geq u_{\mathcal{R}}\left(\tilde{\theta}^{\mathcal{R}}, \boldsymbol{\theta}^{\mathcal{W}}\right)$.

- Individual Rationality (IR): A mechanism is IR if each truthful mobile user has a non-negative expected utility, i.e., $u_{i}\left(\theta_{i}, \boldsymbol{\theta}_{-i}, \theta^{\mathcal{R}}\right) \geq 0$ and $u_{\mathcal{R}}\left(\theta^{\mathcal{R}}, \boldsymbol{\theta}^{\mathcal{W}}\right) \geq 0$.

- Economic Efficiency (EE): The EE property is satisfied if the mechanism $\mathcal{M}$ achieves the highest social welfare as in (1) when all mobile users are truthful. In fact, if truthful information can be obtained from $\mathcal{R}$ and $\mathcal{W}$, EE is immediately achieved at maximum expected utility.

- Budget Balance (BB): BB indicates that the sum of mobile users' payment is 0, i.e., $\sum_{i}^{N} r_{i}+r_{\mathcal{R}}=0$. A mechanism is weak $\mathrm{BB}$ if $\sum_{i}^{N} r_{i}+r_{\mathcal{R}} \geq 0$. Usually, weak BB indicates monetary transfer in the form of taxes between the mobile users and the mechanism $\mathcal{M}$.

\section{FAult-Tolerant InCEntivis ation FOR Mobile P2P CROWD SERVICE}

In this section, we present the fault-tolerant incentivisation mechanism (FTIM) for MPCS systems, which is developed based on the fault-tolerant mechanism design (FTMD) framework [19] and considers the possibility of untruthful requester. Two payment strategies for FTIM are proposed to satisfy mechanism properties under different application scenarios.

\section{A. Fault-Tolerant Incentivisation Mechanism (FTIM)}

As shown in [19], a fault-tolerant mechanism incorporating the execution outcomes of a task $\mathcal{T}$ follows two major steps:

1) Social welfare maximisation: For a task, find the worker with the highest expected social welfare:

$$
\alpha=\underset{i \in \mathcal{W}}{\arg \max }\left\{\tilde{p}_{i} \cdot \tilde{V}-\tilde{c}_{i}\right\},
$$

where $\tilde{V}, \tilde{p}_{i}$ and $\tilde{c}_{i}$ are the declared types. Similarly, the worker $\mathcal{W}_{\beta}$ who contributes the second highest social welfare can be found:

$$
\beta=\underset{i \neq \alpha \in \mathcal{W}}{\arg \max }\left\{\tilde{p}_{i} \cdot \tilde{V}-\tilde{c}_{i}\right\}
$$

with declared completion probability $\tilde{p}_{\beta}$ and cost $\tilde{c}_{\beta}$.
2) Contingent payment: The payment strategy for worker $\alpha$ is conditional on the completion of the assigned task [19]:

$$
r_{\alpha}= \begin{cases}\tilde{V}-\left(\tilde{p}_{\beta} \cdot \tilde{V}-\tilde{c}_{\beta}\right) & \text { if the task is completed } \\ -\left(\tilde{p}_{\beta} \cdot \tilde{V}-\tilde{c}_{\beta}\right) & \text { otherwise }\end{cases}
$$

where the negative sign of $r_{\alpha}$ indicates making a payment (penalty). Since the $r_{\alpha}$ is independent of types of $\mathcal{W}_{\alpha}$, $\mathcal{W}_{\alpha}$ and similarly $\mathcal{W}_{\beta}$ can be considered as truthful, thus $\tilde{p}_{\beta} \equiv p_{\beta}$ and $\tilde{c}_{\beta} \equiv c_{\beta}$. The expected utility for $\mathcal{W}_{\alpha}$ is:

$$
u_{\alpha}=\mathbf{E}\left(r_{\alpha}\right)-c_{\alpha}=p_{\alpha} \cdot \tilde{V}-p_{\beta} \cdot \tilde{V}+c_{\beta}-c_{\alpha}
$$

As for the requester $\mathcal{R}$, the payment strategy is:

$$
r_{\mathcal{R}}= \begin{cases}-\left[\tilde{V}-\left(\tilde{p}_{\beta} \cdot \tilde{V}-\tilde{c}_{\beta}\right)\right] & \text { if the task is completed } \\ \tilde{p}_{\beta} \cdot \tilde{V}-\tilde{c}_{\beta} & \text { otherwise }\end{cases}
$$

with expected utility $u_{\mathcal{R}}\left(\tilde{p}_{\beta} \equiv p_{\beta}, \tilde{c}_{\beta} \equiv c_{\beta}\right)$ :

$$
u_{\mathcal{R}}=p_{\alpha} \cdot V-\mathbf{E}\left(r_{\mathcal{R}}\right)=p_{\alpha} \cdot V-p_{\alpha} \cdot \tilde{V}+p_{\beta} \cdot \tilde{V}-c_{\beta}
$$

where $V$ is the true value of task $\mathcal{T}$ which is only known by requester $\mathcal{R}$. This FTMD satisfies IC, IR (for both $\mathcal{W}_{\alpha}$ and $\mathcal{R}$ ), and EE, given that requester $\mathcal{R}$ is truthful, i.e., $\tilde{V}=V$.

However, the requester $\mathcal{R}$ cannot be guaranteed as a truthful agent in MPCS systems. As shown in (7), the expected utility $u_{\mathcal{R}}$ for $\mathcal{R}$ is dependent on its declared type $\tilde{V}$. Therefore, $\mathcal{R}$ can act strategically to improve its utility by declaring a $\tilde{V}<$ $V$, thus violating the IC property. As a result, FTMD cannot be applied to MPCS systems if we need an IC mechanism. Nevertheless, one can clearly see that EE is preserved with maximised social welfare and IR is maintained for all agents including $\mathcal{R}$. What is more, since $E\left(r_{\mathcal{R}}+r_{\alpha}\right)=0$, BB is satisfied and there is no monetary transfer between the mobile users (workers $\mathcal{W}$ and requester $\mathcal{R}$ ) and the mechanism $\mathcal{M}$. In summary, by directly applying FTMD to MPCS system, the mechanism gains a new property $\mathrm{BB}$ by sacrificing IC. This phenomenon can be well explained by Theorem III.1.

Theorem III.1. There is no social choice function (mechanism) that is truthfully implementable (IC) and meanwhile is individual rational (IR), efficient (EE) and weak budget balanced (weak $B B$ ).

Proof. A similar proof is given for a general mechanism in [17], [18]. We can also show that it is true for any mechanisms in MPCS systems. We define the expected payment received by the requester as $x$, the payment received by $\mathcal{W}_{\alpha}$ is $y$ when task is completed and $-z$ when failed. Thus $u_{\mathcal{R}}=p_{\alpha} V+x$ and $u_{\alpha}=p_{\alpha} y-\left(1-p_{\alpha}\right) z-c_{\alpha}$. To satisfy IR, $u_{\mathcal{R}} \geq 0$ and $u_{\alpha} \geq 0$. For both EE and weak $\mathrm{BB}, x+p_{\alpha} y-\left(1-p_{\alpha}\right) z \leq 0$. We plot the feasible region for $\mathrm{y}$ and $\mathrm{z}$ given these inequalities in Fig. 2, in which the shaded area indicate the feasible region for $y$ and $z$ and $x$ is chosen between $-V$ and $-c_{\alpha}$. In order to have IC, $x, y$ and $z$ must be constants. However, given all possible values for $V$ and $c_{\alpha}$, it is possible that the area of the feasible region in Fig. 2 is zero, thus limiting $y$ and $z$ on the line 


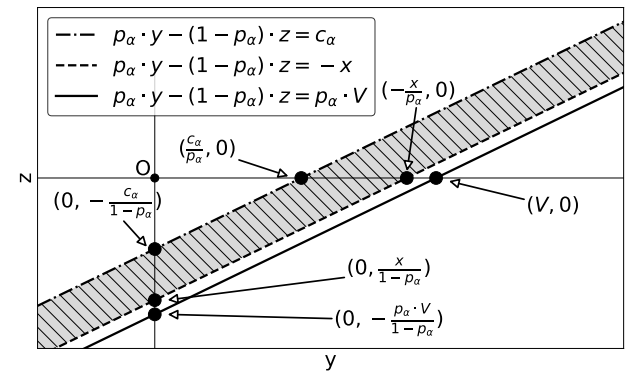

Fig. 2. Feasible regions with IR, EE and weak BB in proof for Theorem III.1

$p_{\alpha} y-\left(1-p_{\alpha}\right) z-c_{\alpha}=0$ and forces either $y$ or $z$ to be dependent on $c_{\alpha}$, which contradicts the IC property. Therefore, for MPCS systems, it is not possible to design a mechanism satisfying IC, IR, EE and (weak) BB at the same time.

\section{B. Proposed Scenario Based Payment Strategies for FTIM}

As indicated by Theorem III.1. we need to drop one of the four properties among IC, IR, EE, and BB. In this paper, we consider two potential application scenarios for MPCS systems which require different mechanism properties and propose payment strategies for each scenario. The scenarios and their desired mechanism properties are introduced as follows:

a) Scenario\#1: In Scenario\#1, the roles of mobile crowd workers are played by individual mobile users. To incentivise crowd workers to participate, it is a must to satisfy the property IR. Moreover, IC must also be satisfied by a truthfully implementable mechanism and thus EE. Therefore, we aim to achieve IC, IR and EE and decide to sacrifice BB in this scenario. We argue that the potential MPCS operators are incentivised to subsidise mobile users to provide and use services because they are able to earn revenues from monetising acquired data and bring down costs of other business, e.g. mitigating cellular network congestions.

b) Scenario\#2: As for Scenario\#2, mobile crowd workers are not individual mobile users any more, but rather mobile nodes (mobile base stations, access points or drones) operated by content providers and Internet service providers (ISP). These providers are spontaneously offering services and data to their customers. As a result, the IR property for mobile workers can be dropped. However, due to potential competition among different service providers, IC must be maintained for crowd workers and overall; the required properties in Scenario\#2 are IC, IR (requester only), weak BB and EE.

1) Payment strategy for Scenario\#1: Assume that worker $\mathcal{W}_{\alpha}$ is assigned the task, the payments $\mathcal{W}_{\alpha}$ and $\mathcal{R}$ should make conditional on task execution results under Scenario\#1 are:

$$
\begin{aligned}
& r_{\alpha}= \begin{cases}\tilde{V}-\left(p_{\beta} \cdot \tilde{V}-c_{\beta}\right) & \text { if task is completed } \\
-\left(p_{\beta} \cdot \tilde{V}-c_{\beta}\right) & \text { otherwise }\end{cases} \\
& r_{\mathcal{R}}= \begin{cases}-\tilde{V} & \text { if task is completed } \\
\frac{p_{\alpha}}{p_{\beta}} \cdot \frac{p_{\beta} \cdot \tilde{V}-c_{\beta}}{1-p_{\alpha}} & \text { otherwise }\end{cases}
\end{aligned}
$$

As proved in [19], the expected utility $u_{\alpha}$ for worker $\alpha$ is larger than 0 . The expected utility of the requester $\mathcal{R}$ is:

$$
u_{\mathcal{R}}=p_{\alpha} \cdot V+\mathbf{E}\left(r_{\mathcal{R}}\right)=p_{\alpha} \cdot V-\frac{p_{\alpha}}{p_{\beta}} c_{\beta} \geq 0
$$

Therefore, this payment strategy satisfies IR. As shown in 10 , the expected payment of $\mathcal{R}$ is not dependent on its private type $\tilde{V}$ and a benign $\mathcal{R}$ will thus reveal its true type. Based on this and (5), the expected utility for worker $\alpha$ becomes:

$$
u_{\alpha}=p_{\alpha} \cdot V-p_{\beta} \cdot V+c_{\beta}-c_{\alpha},
$$

Moreover, since the payment made by $\mathcal{W}_{\alpha}$ is independent of its type, IC is satisfied for both $\mathcal{R}$ and $\mathcal{W}_{\alpha}$.

The utility achieved among all mobile users is thus:

$$
\begin{aligned}
u & \left.=u_{\alpha}+u_{\mathcal{R}}=11\right]+10 \\
& =p_{\alpha} \cdot V-c_{\alpha}+\frac{p_{\alpha}-p_{\beta}}{p_{\beta}}\left(p_{\beta} \cdot V-c_{\beta}\right),
\end{aligned}
$$

Note that since $p_{\beta} \cdot V-c_{\beta} \geq 0$, the utility can be larger or smaller than the maximum social welfare $p_{\alpha} \cdot V-c_{\alpha}$ given different $p_{\alpha}$ and $p_{\beta}$. When $p_{\alpha} \leq p_{\beta}, u \leq p_{\alpha} \cdot V-c_{\alpha}$ and the mechanism effectively levies a tax $\left(p_{\beta}-p_{\alpha}\right) \cdot\left(p_{\beta} \cdot V-c_{\beta}\right) / p_{\beta}$ from the mobile users. In this case, weak BB is achieved. However, when $p_{\alpha} \geq p_{\beta}, u \geq p_{\alpha} \cdot V-c_{\alpha}$ and the mechanism has to subsidise the mobile users by $\left(p_{\alpha}-p_{\beta}\right) \cdot\left(p_{\beta} \cdot V-c_{\beta}\right) / p_{\beta}$. In summary, as presented and proved, this payment strategy for Scenario\# 1 achieves IC, IR and EE and only partially achieves weak $\mathrm{BB}$ conditional on $p_{\alpha} \leq p_{\beta}$.

2) Payment strategy for Scenario\#2: The objective of payment strategy for Scenario\#2 is to achieve IC, EE, (weak) BB and IR for requester $\mathcal{R}$. As shown in (8) and (9) of Scenario\#1, when $p_{\alpha} \leq p_{\beta}$, the strategy satisfies all four properties. Therefore, in this part, we only focus on designing the scenario for the case $p_{\alpha} \geq p_{\beta}$ and we propose the following payment strategy for worker $\alpha$ when $p_{\alpha} \geq p_{\beta}$ :

$$
r_{\alpha}^{p_{\alpha} \geq p_{\beta}}=c_{\beta} \text {, for both cases }
$$

and its expected utility is therefore:

$$
u_{\alpha}^{p_{\alpha} \geq p_{\beta}}=c_{\beta}-c_{\alpha},
$$

Note that the worker will have a negative utility if $c_{\beta}<c_{\alpha}$. Since the payment is independent of its type, the worker will truthfully reveal its type $p_{\alpha}$ and $c_{\alpha}$ and achieve IC. As for requester $\mathcal{R}$, the payment is the same as in Scenario\#1:

$$
r_{\mathcal{R}}^{p_{\alpha} \geq p_{\beta}}= \begin{cases}-\tilde{V} & \text { if task is completed } \\ \frac{p_{\alpha}}{p_{\beta}} \cdot \frac{p_{\beta} \cdot \tilde{V}-c_{\beta}}{1-p_{\alpha}} & \text { otherwise }\end{cases}
$$

and its expected utility is the same as $(10)$. As already proved, IC and IR are both achieved for $\mathcal{R}$ with this strategy. Therefore, the total utility for all mobile users under Scenario\#2 is:

$$
\begin{gathered}
u^{p_{\alpha} \geq p_{\beta}}=u_{\alpha}^{p_{\alpha} \geq p_{\beta}}+u_{\mathcal{R}}^{p_{\alpha} \geq p_{\beta}}=10 \\
=p_{\alpha} \cdot V-c_{\alpha}+\frac{p_{\beta}-p_{\alpha}}{p_{\beta}} \cdot c_{\beta},
\end{gathered}
$$


Since $p_{\alpha} \geq p_{\beta}$, (16) takes a tax from mobile users. Combining (16) and (12), one can show that although the payment strategy cannot guarantee IR for workers, it achieves the required weak BB for Scenario\#2.

\section{MPCSTOKEn: SMART CONTRACT ENABLED INCENTIVISATION AND PAYMENT SETTLEMENT}

The incentivisation mechanism FTIM proposed in section III offers a number of desirable properties, however, there are still several practical challenges of building FTIM into a real-world MPCS system:

a) Transparency and decentralisation of the mechanism: Although the mechanism designer is usually considered trustful in implementing the mechanism, it would be of the mobile users' interest to verify the details of the mechanism and avoid the failure from the mechanism designer. Moreover, as a distributed service, decentralisation of the function for mechanism designer is also important.

b) Counter-party risk: The tasks in MPCS systems are conducted off-line and the payment is contingent on the task execution results, however, there is no definitive approach which can be taken by the mechanism designer to verify the task execution results in order to enforce the payment.

c) Implementation and operation cost: Implementing and operating an incentivisation mechanism incur cost, especially the financial cost associated with frequent and fragmentary payment in a distributed system. Reducing and optimising the relevant cost would add huge value to the practicality of the mechanism and MPCS systems.

In this part, we firstly present a blockchain/smart contract enabled system architecture for FTIM to offer the required transparency and decentralisation of the mechanism. Additionally, to mitigate counter-party risk, we explore a micropayment mechanism by making payments on a per micro-task basis, in which payment channels are used for reducing the cost associated with payment settlement. In the end, cost for deploying and operating smart contracts are presented and related optimisation methods are discussed.

\section{A. Smart Contract Enabled FTIM}

Fig. 3 illustrates the proposed architecture for a smart contract enabled MPCS system. In general, the system performs two categories of operations - on-chain and off-chain. Onchain indicates that the operations will be confirmed by the blockchain and cannot be revoked thereafter, while off-chain operations are carried out in the off-line mobile P2P network between mobile devices and are pending any real transactions or payments on blockchain. In this paper, we propose a smart contract based approach to facilitate on-chain operations and assist off-chain operations performed by mobile applications. In the following parts, we show the functionalities offered by the smart contract - MPCSToken contract as well as how it could be used to interact with the off-chain functions provided by a mobile application.

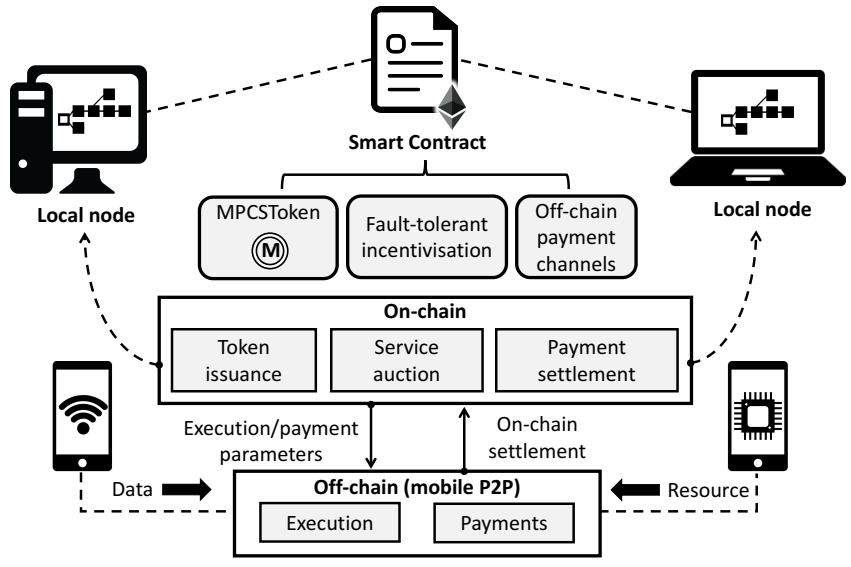

Fig. 3. Architecture of smart contract enabled incentivisation for MPCS systems.

TABLE I

IMPLEMENTED ABI OF MPCSTOKEN CONTRACT

\begin{tabular}{cc}
\hline \hline ABI & Description \\
\hline \hline MPCSToken () & Contract constructor \\
\hline transfer () & Transfer token from one account to another \\
\hline getBalance () & Get the balance of an account \\
\hline contractDestroy () & Destroy the deployed contract \\
\hline auctionStart () & $\begin{array}{c}\text { Requester starts an auction by initialising } \\
\text { relevant variables in the contract }\end{array}$ \\
\hline auctionFinish () & $\begin{array}{c}\text { Requester finishes an auction whereby the } \\
\text { contract computes the maximum system utility } \\
\text { and assigns the task to the winning worker }\end{array}$ \\
\hline getAuctionState () & $\begin{array}{c}\text { Get state of an auction, such as the number of } \\
\text { workers, winning worker and auction results }\end{array}$ \\
\hline report () & Report types of a worker \\
\hline getWorkerState () & $\begin{array}{c}\text { Get the types of a worker, including worker } \\
\text { cost, and task execution success rate }\end{array}$ \\
\hline channelPayin () & Set up a payment channel by paying deposit \\
\hline channelPayout () & Close payment channel by settling payments \\
\hline channelTimeout () & Close payment channel after timeout \\
\hline \hline
\end{tabular}

1) MPCSToken contract: Recently, token-based smart contracts have been widely adopted by decentralised applications (DApp) on Ethereum blockchain [20]. There are many benefits of implementing a token-based contract. For example, it enables DApp developers to produce and define their own cryptocurrencies, which gives the developers more freedom in account and payment management. Moreover, DApp developers can launch Initial Coin Offering (ICO) to finance the development and operation of the DApp. Similar to other token contracts, the MPCSToken contract issues a fixed amount of MPCSToken and each user in the MPCS system owns an account in the contract for making payment using MPCSToken. In addition, MPCSToken contract implements the payment strategies of FTIM, which involves a number of functions such as service auction for revealing users' private types and payment settlements. The functions (called Application Binary Interfaces (ABI) in Ehereum [21]) provided by MPCSToken are shown in Table 1 . Note the details of payment channels will be discussed in Section IV-B

2) Mobile application: As shown in Fig. 3, a mobile application is used to handle task execution functions and generate 
payment receipts between mobile users in an off-line fashion. The requester can use the application to advertise its task request and initiates an auction to reveal workers' parameters. After the MPCSToken contract confirms the allocation, the task will be executed by the worker and payment proofs are generated and exchanged between mobile users. These offchain operations can be enabled using different short range communication technologies such as Bluetooth and WiFiDirect and run on any mobile platform. However, the detailed design should be made application specific, so we do not go into application details in this paper.

Using the ABI provided by the MPCSToken contract and the mobile application, requesters can find crowd workers to execute tasks and settle payments by following the steps below:

1) Requester $\mathcal{R}$ advertises its task request to nearby mobile workers. The request contains a certain number of requirements the crowd worker should satisfy. The requester also starts an auction on the MPCSToken contract and reports its value for the task by calling auctionStart() ABI.

2) After receiving the request, workers start reporting their types to the MPCSToken contract such as their cost and execution probability through report() ABI.

3) The auction finishes after receiving a notification from the requester (auctionFinish()). The contract computes the highest expected social welfare as in (2) and records the allocation result as well as reported types.

4) Both the workers and the requester are notified of the task allocation decision and the winning worker and the requester start a payment channel in the MPCSToken contract using the channelPayin() ABI. After tasks are executed, the worker and requester exchange execution results and payment proofs based on the scenario that is adopted by the specific application and corresponding payment strategies as indicated by (8), (9) and (13).

5) The requester and worker can send the proofs to the MPCSToken contract to settle payments by calling channelPayout().

\section{B. Micropayment Mechanism}

As aforementioned, in MPCS systems, the mechanism is not able to verify the outcome of the off-line task execution results. Therefore, the requester or the worker can become insolvent without committing to the execution result. In order to mitigate this risk, we propose a micropayment mechanism to settle fragmentary payments on a per micro-task basis.

1) Micro-tasks: In most mobile $\mathrm{P} 2 \mathrm{P}$ crowd service, the values obtained from task executions are commonly noncombinatorial, i.e., $v\left(\sum_{j}^{M} \mathcal{T}_{j}\right)=\sum_{j}^{M} v\left(\mathcal{T}_{j}\right)$. For example, for an Internet sharing task, if some network transaction is half finished, the requester can still resort to other communication methods to continue to consume network resources. A number of approaches have also been proposed recently to avoid wasting of network data during interruption [22]-[25]. Computation offloading tasks also have non-combinatorial values.
For example, offloaded image processing tasks can be executed on a per-image basis [9] and the remaining images can later be processed locally or by another worker.

We therefore consider that a single task $\mathcal{T}$ has a number of micro-tasks $\mu(\mathcal{T})_{k}$, where $k \in\{1,2, \ldots, K\}$. Assume that the execution of each $\mu(\mathcal{T})_{k}$ is independent and follows the same distribution for its successful rate. Such an assumption is valid since the transactions in MPCS systems are short-lived and the factors affecting the execution quality is relatively stable over this short time period. Moreover, $\mu(\mathcal{T})_{k}$ for the same $\mathcal{T}$ usually have homogeneous features and quality requirements. Based on this, for each task, the outcome $X_{k}$ of executing $\mu_{k}(\mathcal{T})$ follows a Bernoulli distribution $\operatorname{Bernoulli}(p)$ :

$$
\operatorname{Pr}\left(X_{k}\right)= \begin{cases}p & \text { if } X_{k}=1 \\ 1-p & \text { if } X_{k}=0\end{cases}
$$

Therefore, the expected utility for executing $K$ micro-tasks $\mu_{k}(\mathcal{T})$ in $\mathcal{T}$ can be presented as:

$$
\begin{aligned}
& E\left[v\left(\sum_{k=1}^{K} X_{k} \cdot \mu(\mathcal{T})_{k}\right)\right]=E\left[\sum_{k=1}^{K} v\left(X_{k} \cdot \mu_{k}(\mathcal{T})\right)\right]= \\
& \sum_{k=1}^{K} E\left[X_{k} \cdot v\left(\mu_{k}(\mathcal{T})\right)\right]=\sum_{k=1}^{K} v\left(\mu_{k}(\mathcal{T})\right) \cdot E\left[X_{k}\right]= \\
& \sum_{k=1}^{K} p \cdot v\left(\mu(\mathcal{T})_{k}\right)=p \cdot v(\mathcal{T})
\end{aligned}
$$

As shown in (18), the probability $p$ for completing each microtask is in fact the probability $p_{i}$ that worker $i$ successfully executes task $\mathcal{T}$.

2) Micropayment and Payment Channels: Based on (18), a payment can also be made on the micro-task level. Such microtask based payments (micropayment) are able to mitigate any associated counter-party risk. However, if the payment is settled per micro-task, there will be many payment settlement transactions between the mobile users and the contract. Such frequent settlements may cause a large overhead and financial cost to the system. In this paper, we propose using payment channels to reduce this overhead. A payment channel is a class of techniques that allow users to make multiple transactions without committing the transactions to blockchain [26]. The concept of payment channels appeared as an improvement to the blockchain for micropayment cost reduction [26]-[28]. The protocols and systems for handling micropayments using Bitcoin blockchain in the mobile environment has been studied in our previous work [29] and the payment channels for Ethereum in MPCS systems can be implemented by following a very similar protocol.

Fig. 4 shows how micro-tasks and the payments are made between a requester $\mathcal{R}$ and a worker $\mathcal{W}$. Firstly, two payment channels (multisig accounts) are created for $\mathcal{R} \mapsto \mathcal{W}$ channel and $\mathcal{W} \mapsto \mathcal{R}$ channel and $\mathcal{R}$ and $\mathcal{W}$ put some deposits into these accounts respectively. $\mathcal{R}$ sends micro-tasks to $\mathcal{W}$, who then tries to execute and return the results to $\mathcal{R}$. If a micro-task is successfully executed, a payment message is sent from $\mathcal{R}$ 


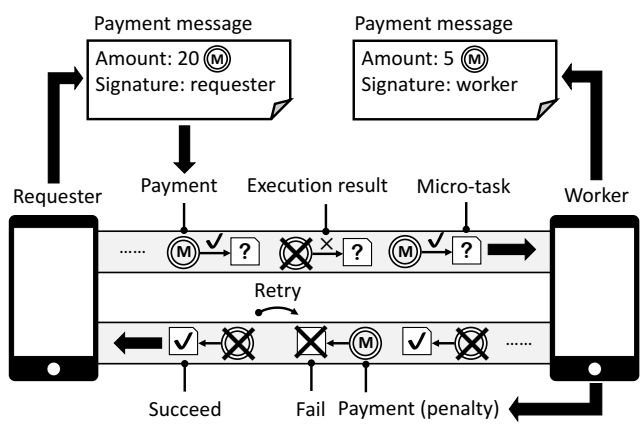

Fig. 4. Micro-task execution and micropayments.

to $\mathcal{W}$. The message contains information such as the payment amount and signed by $\mathcal{R}$. Note that the amount specified in the message is the accumulated amount, which is the up-to-date accumulated payment $\mathcal{R}$ owes to $\mathcal{W}$ based on FTIM. Similarly, a payment message containing the accumulated penalty is signed and sent to $\mathcal{R}$ by $\mathcal{W}$ after every failed micro-task. Note that according to FTIM, $\mathcal{R}$ only sends payment to $\mathcal{W}$ when the task is successful and $\mathcal{W}$ only pays $\mathcal{R}$ (penalty) if the task has failed. In this way, all payments are made off-chain and not realised by the mechanism (smart contract). Later, When $\mathcal{R}$ or $\mathcal{W}$ decides to confirm received payments, it is able to sign the received payment message and sends it to the smart contract on blockchain to claim the funds deposited in the account of either $\mathcal{R} \mapsto \mathcal{W}$ or $\mathcal{W} \mapsto \mathcal{R}$ channel respectively. The contract can check if the payments have been made in accordance with the payment strategy and then credit one's accounts by making token transfers from the multisig accounts.

\section{Cost of Smart Contract}

Using a public blockchain is expensive, especially with frequent access to the blockchain storage. Therefore, optimisation should be done on the smart contract to lower the cost of using it. One approach is to move some operations off the chain, for example, the payment channels effectively move some onchain operations off-chain therefore reducing the number of messages exchanged between the contract and mobile users. Another direction is to optimise the contract code and to use cheap operations to realise the same program logic [30].

The most important performance metric of the Ethereum smart contracts is gas cost. Gas is used to calculate the transaction fees paid to miners for using the computation and storage resources on the Ethereum blockchain. The fees related to the consumed gas are computed by multiplying amount of gas by the gas price, which has a unit of wei or Gwei and is directly related to ETH, the cryptocurrency for the Ethereum blockchain $\left(1 \mathrm{ETH}=10^{18}\right.$ wei, Gwei $=10^{9}$ wei $)$. When a smart contract executes code or uses blockchain storage, a fee will be consumed and paid by the code or storage users. At the time of writing (Jan 2018), the average gas price is 1 Gwei and the median transaction confirmation time with this gas price is 1 minute 1 Therefore, in the following parts, we use 1 Gwei

\footnotetext{
${ }^{1}$ Although higher gas price indicates faster transaction confirmation, a low gas price such as 1 Gwei can also achieve fast confirmation [31
}

TABLE II

EXPENSIVE OPERATIONS ON ETHEREUM BLOCKCHAIN

\begin{tabular}{cccc}
\hline \hline Name & Description & Gas & $\$$ Fee \\
\hline \hline$G_{\text {create }}$ & Contract creation & 32000 & 0.015 \\
\hline$G_{\text {transaction }}$ & Paid for every transaction & 21000 & 0.01 \\
\hline$G_{\text {sset }}$ & Storage set to non-zero from zero & 20000 & 0.01 \\
\hline$G_{\text {sreset }}$ & $\begin{array}{l}\text { Storage zeroness remains } \\
\text { unchanged or set to zero }\end{array}$ & 5000 & 0.0025 \\
& & \\
\hline$G_{\text {callvalue }}$ & Paid for a non-zero value transfer & 9000 & 0.005 \\
\hline$G_{\text {newaccount }}$ & Paid for account creation & 25000 & 0.05 \\
\hline \hline
\end{tabular}

as the gas price when calculating Ethereum fees. In addition, to give readers a sense of corresponding monetary value, we also show the dollar value of those fees based on the 6-month average ETH price of $\$ 487$.

Table. II shows the most expensive operations in terms of gas cost and fee. Among these operations, $G_{\text {callvalue }}$ and $G_{\text {newaccount }}$ are not used by the MPCSToken contract since the account and payment transfer is managed by the contract internally. Instead, these operations consume gas by $G_{\text {sset }}$ (account creation) and $G_{\text {sreset }}$ (value transfer). $G_{\text {create }}$ is used to create the contract and is called only once. Therefore, the main sources of gas consumption for the MPCSToken contract are $G_{\text {transaction }}$ (number of messages sent to blockchain for each task execution in MPCS systems), and $G_{\text {sset }}$ and $G_{\text {sreset }}$ (store/reset a value in the contract). One should also note that if a same user repeatedly participates in a task, the storage related to that user may be set and reset frequently. In order to reduce fees, reusing the same storage after resetting it to zero $\left(G_{\text {sset }}\right)$ after usage would increase the gas cost by 15000 compared to directly modifying the storage without resetting it to zero $\left(G_{\text {sreset }}\right)$. Such techniques would be extremely important when building the contract code.

\section{Evaluation}

We build the MPCSToken smart contract on Ethereum blockchain. The smart contract implements all aforementioned ABIs for payment strategies and the micropayment mechanism. Moreover, a mobile data offloading application is developed and used to conduct a real-world MPCS based offloading experiment. We evaluate the performance of the MPCSToken contract in terms of 1) blockchain performance such as the cost of ABI calls and transactions, 2) performance of the MPCS prototype system in a real-world experiment, 3) the economic impact in large scale social networks.

\section{A. MPCS Prototype Implementation}

The prototype consists of the MPCSToken smart contract and a D2D mobile data offloading application:

1) MPCSToken prototype: The MPCSToken contract is built on the Ethereum blockchain using the programming language Solidity [32]. The deployed contract on Ropsten Testnet can be found at [33]. The ABIs the contract offers are shown in Table I Some of these ABIs are getters and thus calling them does not consume gas. Moreover, some ABI are called a limited number of times during the lifetime of the 
TABLE III

GAS USAGE AND FEE (USD) OF ABI CALLS FOR THE MPCSTOKEN CONTRACT

\begin{tabular}{ccccc}
\hline \hline ABI & First gas $(\$)$ & Subsequent gas $(\$)$ & $\mathcal{R}$ & $\mathcal{W}$ \\
\hline \hline auctionStart () & $103158(0.04)$ & $43158(0.02)$ & $\checkmark$ & \\
\hline report () & $91793(0.04)$ & $46793(0.02)$ & & $\checkmark$ \\
\hline auctionFinish () & $37942(0.01)$ & $37942(0.01)$ & $\checkmark$ & \\
\hline channelPayin () & $89455(0.04)$ & $59455(0.02)$ & $\checkmark$ & $\checkmark$ \\
\hline channelPayout ()$\sim 65500(0.03)$ & $\sim 50500(0.02)$ & $\checkmark$ & $\checkmark$ \\
\hline \hline
\end{tabular}

contract. For example, the contract constructor is called only once while transfer () is called only when the token is issued by the contract owner (MPCS service operator). In the long run, these costs would converge to zero on a per transaction basis, therefore, in terms of the cost for using this contract, only these ABI in Table. III should be considered. We also indicate in Table. III that whether one ABI will be called by requester $\mathcal{R}$ or the winning worker $\mathcal{W}$ when executing a task.

As shown in Table [III, the cost for the ABI is different between first and subsequent calls made by the same requester. The difference arises from the different gas usage of $G_{\text {sset }}$ and $G_{\text {sreset }}$, which is always a multiple of 15000 . For example, the difference of 60000 gas for auctionStart() is caused by using $G_{\text {sset }}$ or $G_{\text {sreset }}$ to initialise four parameters of the requester for an auction including the number of workers, start time, timeout and the task value. Only the first call by a requester to initialise these parameters use $G_{s s e t}$ while the subsequent calls use $G_{\text {sreset }}$, which consumes 15000 less than $G_{\text {sset }}$. As for report (), less gas will be consumed if the number of workers in the current auction is smaller than the maximum number of workers participating in previous auctions of the same requester. For example, if the maximum number of workers ever participated in the auction initiated by a requester is five, then as long as the number of workers in the current auction is smaller than five, the cost of calling report () is 46793. Generally speaking, the higher cost associated with the first call will be averaged out by subsequent calls when there is a sufficient number of ABI calls made by a requester. Furthermore, as seen in Table. III], both requester and worker need to call channelPayin () and channelPayout () since the payment strategy proposed by FTIM is bi-directional and requires deposits from both parties. Overall, the average cost is around $\$ 0.08$ for requester and $\$ 0.06$ for the winning worker (the cost for other worker is 0.02) to use the contract for one auction (task), which is an acceptable overhead (compared to PayPal with a fee of $\$ 0.30+[34]$ ) considering the transaction value for tasks in MPCS systems, which we assume could range between $\$ 0.5$ to $\$ 5$.

2) Data offloading mobile application: A data offloading mobile application is implemented for Android OS. The application is able to connect to nearby mobile devices using Bluetooth to download data from a web link. Fig. 5 shows the UI of the mobile app. The requester can use request UI (Fig. 5(a) to configure the link for the data, the value of the task, as well as the number of micro-tasks it wishes
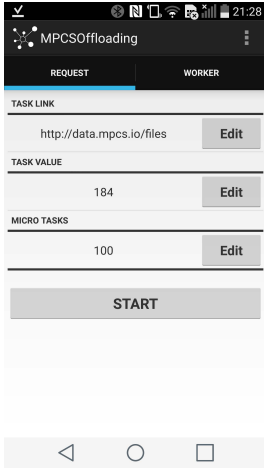

(a) Request UI

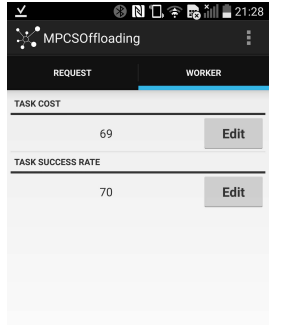

(b) Worker

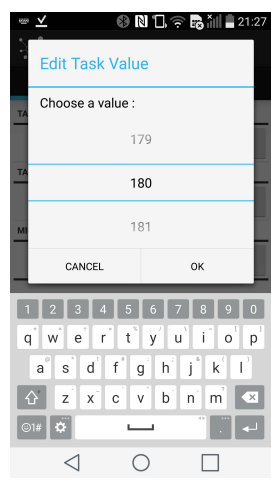

(c) Edit Value UI
Fig. 5. UI of data offloading application.

to split the large task into. Workers can also edit their cost based on their cellular or WiFi network costs (Fig. 5(c) $)$. The task execution probability is automatically generated by the application based on the information of the device's network connectivity and resource availability.

\section{B. Mobile Offloading Experiment}

We set up an experiment using the MPCS prototype system to evaluate the MPCSToken contract performance under Scenario\#1, which represents a fully decentralised service system. In the experiment, we use a fixed data link for the offloading task. Each task has 100 micro-tasks and the task value, task cost and success probability are configured by the individual mobile device. Since in the real world, a local network formed by Bluetooth cannot accommodate a large number of devices, in this experiment, we use six mobile devices and recruit users (college students) to make transactions with each other. Each device has an individual MPCSToken account with an initial balance of 1000. A web server is used to host the blockchain simulator where the MPCSToken contract is deployed. Note that the participating users can adopt different strategies during the experiment such as hiding their devices from the rest of the network (requester only mode), or competing for tasks by connecting to low cost WiFi (worker only mode). The experiment lasts for eight hours and during the experiment, users may lose the access to the service by leaving the experiment area. All the transactions which have taken place during the experiment are recorded.

Fig. 6 shows the balance of participating users' accounts and the contract (mechanism) account. From Fig. 6, one can identify the strategy of each user during the experiment. For example, user\#3 and user\#5 constantly collect more tokens during the experiment and thus act mostly as workers. In fact, their devices have low task costs and thus have won most of the auctions. On the contrary, user\#1 and user\#4 hide themselves from the rest of the network and thus only spend token as requesters for tasks. In addition, other users such as user\#2 and user\#6 experience change of strategies during the experiment. One interesting thing to note is that the balance of the contract (mechanism) increased steadily during the whole experiment. As mentioned in Section III] the payment strategy 


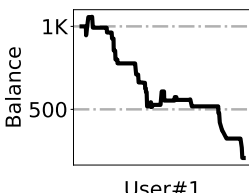

User\# 1

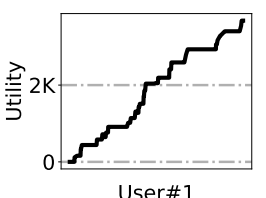

User\# 1

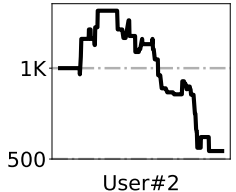

User\#2

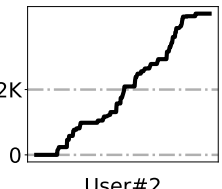

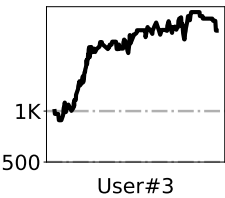

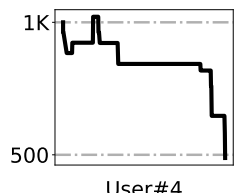

Fig. 6. Balance of participating users.
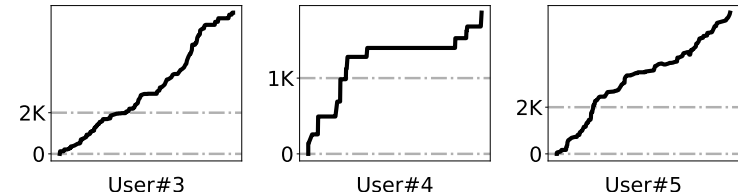

User\# 5

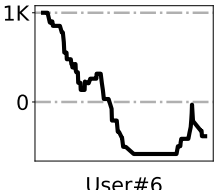

User\#6

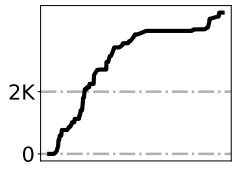

User\#6
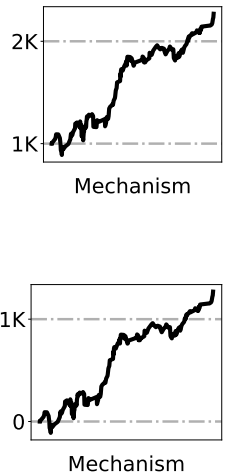

Fig. 7. Utility of participating users.

under Scenario\#l will incur subsidies and thus causing a lower balance for the mechanism if $p_{\beta}<p_{\alpha}$, i.e., a worker with lower execution probability wins. Intuitively this condition will happen more frequently since a worker with higher execution probability is more likely to win. However, in this experiment, workers with smaller $p_{i}$ win since the requesters' task value is close to workers' cost, which favours workers with low costs rather than those with a high execution probability. This result indicates the strong applicability of FTIM in urban areas since the value of the relevant tasks is usually very small and there are a lot of opportunities to get low cost workers due to the population density. Under these conditions, the operator of FTIM is very likely to make a profit and the weak BB property in fact can be well achieved. Fig 7 shows the utility of each user and the mechanism during the experiment, which can further validate the IR property of FTIM.

\section{Simulations}

We evaluate the performance of FTIM and the MPCSToken contract using real-world social contact data. The purpose of the simulations is to evaluate the performance of FTIM 1) considering the impact of human mobility and social contact 2) on a large scale and 3) with different short range communication technologies. Two wireless social networks formed by Bluetooth and WiFi respectively are investigated in the simulations, which are are both obtained from realworld mobility trace data. The Bluetooth social network data [35] have about 40 nodes ${ }^{2}$ and the WiFi data [36] have 20 nodes. Random tasks with each containing 100 micro-tasks are generated by each node every 30 minutes. When a node generates a task, it becomes the requester and assigns a value to the task. Furthermore, each worker is assigned a random cost value between $[0,100]$ and a task execution probability between $[0,1]$ per simulation. We consider all micro-tasks to be homogeneous and has the same probability of success when executed by a worker.

Combining the two datasets and the two payment strategies in Section III, four configurations are evaluated in the simulations. In each configuration, simulations are carried out

\footnotetext{
${ }^{2}$ There are more than 40 nodes in the dataset but only about 40 nodes pro-actively probe and record contacts.
}

100 times and the distributions of the utility of each node are presented in Fig. 8, in which each box plot illustrates the standard deviation, median and range of the node utility. As for Scenario\#1, Fig. 8(a) and 8(b) show that the proposed FTIM ensures that all participating nodes in both networks have positive utility. For Scenario\#2, in which the IR is dropped, although nodes experience negative utility as shown in Fig. 8(c) and 8(d), all nodes tend to have more positive utility than negative ones. As discussed in Section III, a winning worker node receives negative utility under Scenario\#2 when its task execution cost is larger than the worker node achieving the second highest social welfare. However, it is more likely that a winning node's cost is smaller than that of the other nodes, which limits the possibility of negative utility. In addition, as indicated by Fig. 8(d) nodes tend to have fewer negative utilities in the WiFi network compared to the nodes in the Bluetooth network under Scenario\#2. With larger communication range of $\mathrm{WiFi}$, nodes have better access to worker nodes and thus more favourable parameters (i.e., cost, probability) for task execution. Moreover, although the number of nodes in the WiFi network is smaller than the Bluetooth one, the case of negative utility is still lower due to the larger communication range as well. Fig. 9 shows the distributions of the mechanism balance in the simulations. Under Scenario\#1, the mechanism pays more subsidies than taxes, thus incurring a negative balance. The lower balance of WiFi network is the result of a larger number of contacts in its dataset. Since the mechanism only collects taxes under Scenario\#2, the balance of the mechanism is strictly positive for both networks. Fig. 10 shows the percentage of the blockchain fees paid by requesters and workers per transaction in the simulations. For example, requesters pay $191 \mathrm{k}$ Gwei (\$0.08) per transaction for $41 \%$ of all transactions in the Bluetooth network, while in the WiFi network, $90.5 \%$ of the transactions incur a same blockchain fee for the requesters. Overall, the average blockchain fees paid by requesters and workers in the WiFi network are smaller than the Bluetooth network, which can be explained by the smaller number of nodes in the WiFi network since the fee for making ABI calls is dependent on the number of workers in an auction and thus higher fees would be incurred with more nodes in the network. 


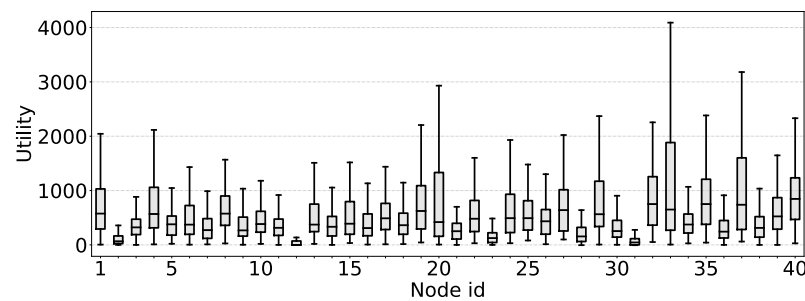

(a) Bluetooth network using payment strategy for Scenario\#1

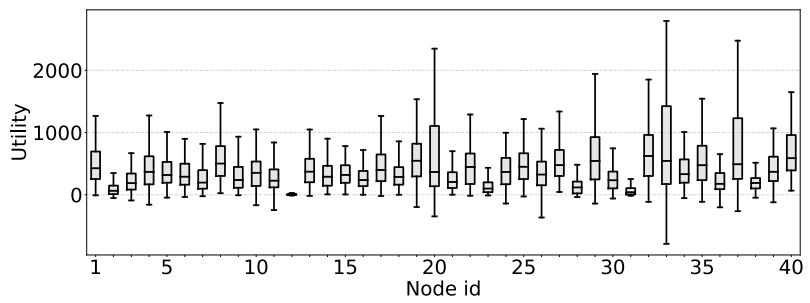

(c) Bluetooth network using payment strategy for Scenario\#2

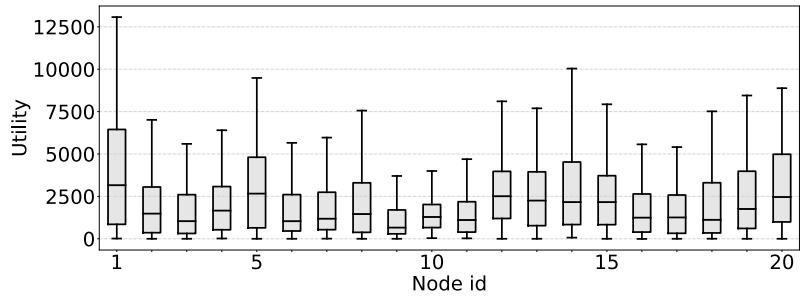

(b) WiFi network using payment strategy for Scenario\#1

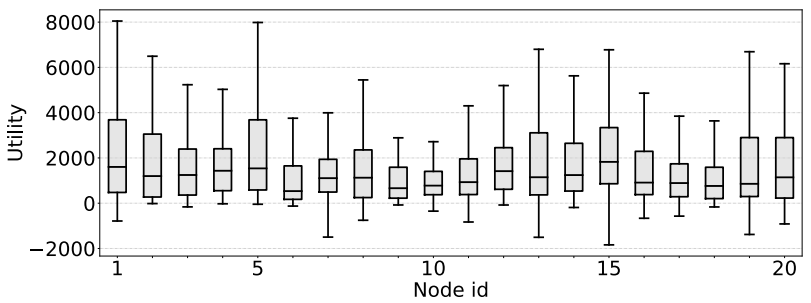

(d) WiFi network using payment strategy for Scenario\#2

Fig. 8. Utility of participating nodes in social networks with different D2D technologies - Bluetooth and WiFi.

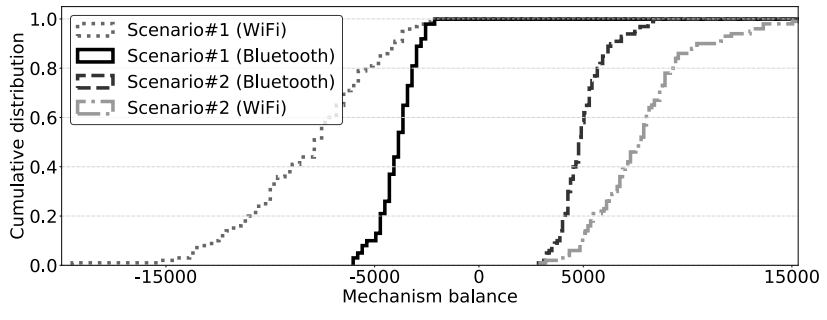

Fig. 9. Mechanism balance under different networks and scenarios.

\section{RELATED WORK}

\section{A. Incentivisation Mechanism for Mobile Crowd Service}

Incentivisation mechanisms have been an important driver for mobile crowd based services. For example, in mobile crowdsensing, auction based mechanism design has been used extensively to discover the truthful cost of crowd workers for collecting data $[11]-[15]$. A fault-tolerant mechanism is also presented to consider the execution uncertainty of data collection tasks [37]. However, these mechanisms usually require the task requester to be trustworthy, which is not a valid assumption in MPCS systems.

Incentivisation mechanisms for $\mathrm{P} 2 \mathrm{P}$ crowd services such as mobile offloading has also become a popular research area recently. Double auction based method is proposed to allocate offloading opportunities through public access points (AP) to mobile network operators (MNO) [16]. However, a double auction requires a large number of bids being placed, while in MPCS systems the number of participants in a transaction is relatively limited. [9] proposes a hidden market based approach to offload computation tasks. A more general mechanism for trading computational resources among a set of nearby mobile devices is proposed in [38]. However, these approaches fail to take into consideration task execution failures, which are very common in mobile P2P networks due to network connectivity issues and the constrained resources

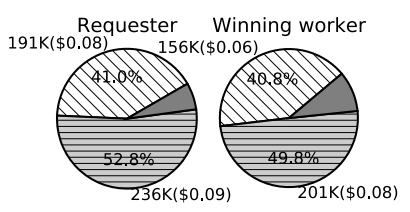

(a) Bluetooth network

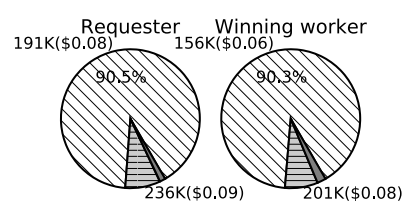

(b) WiFi network
Fig. 10. Distribution of blockchain fee for requester and winning worker.

of mobile devices. Moreover, these researches only focus on theoretical models and lack sufficient contribution to solving important practical issues for incentivisation. As far as we know, this paper is the first work to investigate fault-tolerant incentivisation mechanism for MPCS systems and build a real system to implement such a mechanism.

\section{B. Smart Contract Enabled Computation/Offloading Service}

With the recent development of blockchain, smart contracts can be designed to solve complex problems in a highly decentralised manner. This has brought huge opportunities to apply various incentivisation and trust models to realworld computing problems. For example, smart contracts are employed to avoid untruthful behaviours such as collusion for task offloading in cloud servers [39]. As for mobile systems, [9] proposes a trust model based on cryptocurrency for offloading computation tasks between mobile devices, while [29] builds a trading and payment system for opportunistic data services based on micropayment channels. Compared to this existing work, this paper focuses on practical issues such as data publishing and trading using smart contract to assist incentivisation mechanisms. To our best knowledge, it is the first time that a smart contract has been used to build an incentivisation mechanism for mobile crowd services. 


\section{CONCLUSION}

In this paper, we propose a fault-tolerant incentivisation mechanism (FTIM) for MPCS systems. We highlight two potential application scenarios for MPCS systems and propose two payment strategies respectively which are both conditional on the task execution results. We have provided rigorous proofs to show that these strategies can achieve incentive compatibility, economic efficiency and individual rationality or weak budge balance. Moreover, with the aim to build a practical system, we design a micropayment mechanism to mitigate risk in payment settlements in a decentralised system. We implement the proposed mechanisms as a MPCSToken smart contract and build a mobile application to perform a mobile P2P data offloading experiment. Extensive simulations have also been carried out to investigate the performance on a larger scale. Both the experiment and simulation results indicate that the system achieves excellent performance in improving participants' utility and is also cost effective running on the real-world Ethereum blockchain.

\section{REFERENCES}

[1] D. N. Lane, E. Miluzzo, H. Lu, et al., "A survey of mobile phone sensing," IEEE Commun. Mag., vol. 48, no. 9, pp. 140-150, 2010.

[2] R. K. Ganti, F. Ye, and H. Lei, "Mobile crowdsensing: current state and future challenges," IEEE Commun. Mag., vol. 49, no. 11, pp. 32-39, 2011.

[3] R. Gao, M. Zhao, T. Ye, et al., "Jigsaw: Indoor floor plan reconstruction via mobile crowdsensing," in Proc. ACM Annu. Int. Conf. Mobile Comput. Netw. (MobiCom), 2014, pp. 249-260.

[4] M. Alzantot and M. Youssef, "Crowdinside: Automatic construction of indoor floorplans," in Proc. Int. Conf. Adv. Geograph. Inf. Syst. (SIGSPATIAL), 2012, pp. 99-108.

[5] A. Thiagarajan, et al., "Vtrack: Accurate, energy-aware road traffic delay estimation using mobile phones," in Proc. ACM Conf. Embedded Networked Sensor Syst. (SenSys), 2009, pp. 85-98.

[6] Y. Gao, et al., "Mosaic: A low-cost mobile sensing system for urban air quality monitoring," in Proc. IEEE Conf. Comput. Commun. (INFOCOM), 2016, pp. 1-9.

[7] K. Chen, H. Shen, and H. Zhang, "Leveraging social networks for $\mathrm{p} 2 \mathrm{p}$ content-based file sharing in disconnected manets," IEEE Trans. on Mobile Comp., vol. 13, p. 2, 2014.

[8] M. Chiang, "Mobile peer-to-peer content sharing method and system," 2013, u.S. Patent No. 8,406,206.

[9] D. Chatzopoulos, et al., "Flopcoin: A cryptocurrency for computation offloading," IEEE Trans. on Mobile Comp., vol. 99, p. 1, 2017.

[10] S. Oh, H. Yoo, et al., "Mobile plus: Multi-device mobile platform for cross-device functionality sharing," in Proc. ACM Annu. Int. Conf. Mobile Sys., Apl., and Serv. (MobiSys), 2017.

[11] S. Yang, U. Adeel, and J. McCann, "Backpressure meets taxes: Faithfu data collection in stochastic mobile phone sensing systems," in Proc. IEEE Conf. Comput. Commun. (INFOCOM), 2015, pp. 1490-1498.

[12] J.-S. Lee and B. Hoh, "Sell your experiences: a market mechanism based incentive for participatory sensing," in Proc. IEEE Conf. Perv. Compt. and Comm. (PerCom), 2010, pp. 60-68.

[13] L. Gao, F. Hou, and J. Huang, "Providing long-term participation incentive in participatory sensing," in Proc. IEEE Conf. Comput. Commun. (INFOCOM), 2015, pp. 2803-2811.

[14] T. Luo, H.-P. Tan, and L. Xia, "Profit-maximizing incentive for participatory sensing," in Proc. IEEE Conf. Comput. Commun. (INFOCOM), 2014, pp. 127-135.

[15] T. Luo, S. K. Das, H.-P. Tan, and L. Xia, "Incentive mechanism design for crowdsourcing: An all-pay auction approach," ACM Trans. Intell. Syst. Technol., vol. 7, no. 3, pp. 1-26, 2016.

[16] G. Iosifidis, L. Gao, J. Huang, et al., "A double-auction mechanism for mobile data-offloading markets," IEEE/ACM Trans. Netw. (TON), vol. 23, no. 5, pp. 1634-1647, 2015.
[17] J. Green and L. Jean-Jacques, "Characterization of satisfactory mechanisms for the revelation of preferences for public goods," Econometrica: Journal of the Econometric Society, pp. 427-438, 1977.

[18] L. Hurwicz, "The design of mechanisms for resource allocation," The American Economic Review, vol. 63, pp. 1-30, 1973.

[19] R. Porter, et al., "Fault tolerant mechanism design," Artificial Intelligence, vol. 172, pp. 1783-1799, 2008.

[20] Ethereum tokens, https://www.ethereum.org/token.

[21] Ethereum yellow paper, http://yellowpaper.io/.

[22] Y. Go, Y. Moon, et al., "A disruption-tolerant transmission protocol for practical mobile data offloading," in Proc. ACM Int. Workshop Mobile Opp. Netw. (MobiOpp), 2012, pp. 61-68.

[23] S. Oh, H. Yoo, et al., "Practicalizing delay-tolerant mobile apps with cedos," in Proc. ACM Annu. Int. Conf. Mobile Sys., Apl., and Serv. (MobiSys), 2015, pp. 419-433.

[24] K. Winstein and H. Balakrishnan, "Mosh: An interactive remote shell for mobile clients," in Proc. USENIX Annu. Technical Conf., 2012, pp. 177-182.

[25] R. Mahindra, H. Viswanathan, et al., "A practical traffic management system for integrated lte-wifi networks." in Proc. ACM Annu. Int. Conf. Mobile Comput. Netw. (MobiCom), 2014, pp. 189-200.

[26] Bitcoin payment channels, https://en.bitcoin.it/wiki/Payment_channels.

[27] Lightning Network, https://lightning.network/.

[28] C. Decker and R. Wattenhofer, "A fast and scalable payment network with bitcoin duplex micropayment channels," in Proc. Springer Symp. Self-Stabilizing Sys., 2012, pp. 3-18.

[29] F. Shi, Z. Qin, and J. A. McCann, "Oppay: Design and implementation of a payment system for opportunistic data services," in Proc. IEEE Int. Conf. Distributed Comp. Sys. (ICDCS), 2017, pp. 1618-1628.

[30] T. Chen, X. Li, X. Luo, et al., "Under-optimized smart contracts devour your money," in Proc. IEEE Software Analysis, Evolution and Reengineering (SANER), 2017, pp. 442-446.

[31] ETH gas station, https://ethgasstation.info/.

[32] Solidity, https://solidity.readthedocs.io/.

[33] MPCSToken contract instance, https://ropsten.etherscan.io/address/ 0x4d583a329c8f4cdfc71962c35167d9dc888fb838.

[34] PayPal fees, https://www.paypal.com/us/webapps/mpp/paypal-fees.

[35] Cambridge Haggle Project, http://www.haggleproject.org/.

[36] C. Tuduce and T. Gross, "A mobility model based on wlan traces and its validation," in Proc. IEEE Conf. Comput. Commun. (INFOCOM), 2005, pp. 664-674.

[37] Z. Zheng, Z. Yang, F. Wu, et al., "Mechanism design for mobile crowdsensing with execution uncertainty," in Proc. IEEE Int. Conf. Distributed Comp. Sys. (ICDCS), 2017, pp. 955-965.

[38] X. Wang, X. Chen, and W. Wu, "Towards truthful auction mechanisms for task assignment in mobile device clouds," in Proc. IEEE Conf. Comput. Commun. (INFOCOM), 2017, pp. 1-9.

[39] C. Dong, Y. Wang, A. Aldweesh, et al. "Betrayal, distrust, and rationality: Smart counter-collusion contracts for verifiable cloud computing," in Proc. ACM SIGSAC Conf. on Computer and Communications Security (CSS), 2017, pp. 211-227. 